\title{
STRONG REACHABILITY OF REACTIONS WITH REVERSIBLE STEPS
}

\author{
ESZTER VIRÁGH *1 AND BÁLINT KISS ${ }^{1}$ \\ ${ }^{1}$ Department of Control Engineering and Information Technology, Budapest University of Technology and \\ Economics, Magyar Tudósok krt. 2, Budapest, 1117, HUNGARY
}

\begin{abstract}
The controllability of reactions is an important issue in the chemical industry. The control of reactions is of great practical interest in order to ensure the energy- and time-efficient production of compounds. This paper studies the dynamical models of some chemical reactions in order to verify their controllability with regard to a candidate input signal, namely the change in the ambient temperature of a reaction.
\end{abstract}

Keywords: reversible reaction, strong reachability, controllability, Lie algebra

\section{Introduction}

Chemical reactions are widely applied during the synthesis and transformation processes of organic compounds. The reaction mechanism and resulting products depend mainly on the concentrations of the species, the catalyst used, the ambient temperature, and the ambient pressure. If the values of these parameters are changed, one can obtain different products from the original ones but it is also possible to increase the productivity and energyefficiency of the reaction. Hence the application of a proper feedback law to ensure the latter scenario may be envisaged.

A study of the local controllability by considering the reaction rate coefficient as an input has been presented in Ref. [1]. The authors of Ref. [2] have also considered the reaction rate coefficient as an input and extended the results by claiming that global controllability holds. The controllability of another control input, namely the dilution ratio, is studied in Ref. [3]. General conditions for strong reachability in the case of a temperature input were reported earlier in Ref. [4]. Moreover, the conditions of strong reachability for polymer electrolyte membrane fuel cells (PEMFC), controlled by concentrations, have also been analysed. The motivation to consider the concentrations and temperature (or their rate of change) as input signals is due to the fact that these quantities can be easily modified efficiently by industrial equipment that is currently in use, thus these results can be used as a basis to establish control laws to stabilize a desired reaction performance without major changes being made to the equipment used in production. The oxidation of acetone with hydroxylamine (the oximation reaction) was investigated by Raman spectroscopy [5]. Knowing the mecha-

*Correspondence: viragh.eszter@gmail.hu nism, the controllability is important for this reaction.

Throughout this paper, the candidate variable for control is the rate of change in the temperature $\dot{T}$, i.e. the first time derivative of the ambient temperature. From a practical point of view, this is a simplification since the variable which can be changed externally, denoted by $u$, is not $\dot{T}$ but an algebraic expression involving $u$ and other variables of the system as well. For the dynamics considered in this paper it is always possible, however, to obtain the values of $u$ as a function of $\dot{T}$ and other state variables.

The remaining part of this paper is organized as follows. Sec. 2 briefly revisits the concepts related to the strong reachability of nonlinear dynamical systems and conditions of strong reachability. The differential equations describing the dynamics of reactions are presented so that the rate of change in temperature is considered as the controlled input in Sec. 3. In Sec. 4 the strong reachability of the oximation reaction is studied. The systems in the case of acidic medium in Subsection 4.1 and in weakly basic medium in Subsection 4.2 are analyzed.

In Ref. [4] a sufficient condition for strong reachability was given for reactions with general dynamics. In Sec. 5 , the conditions for strong reachability are given, if the reaction also contains reversible steps. In the last section the conclusions of the paper are drawn.

\section{Study of strong reachability}

Consider the following nonlinear dynamical system, given by the differential equation:

$$
\dot{\xi}=f(\xi)+g(\xi) u, \quad \xi(0)=\xi^{*} \in \mathbb{R}^{n},
$$

where $f, g \in \mathbb{C}^{\infty}\left(\mathbb{R}^{n}, \mathbb{R}^{n}\right)$ are smooth vector fields and $u \in \mathbb{R}$ is the control-input variable. The vector fields 
$f$ and $g$ are often referred to as drift and control vector fields, respectively. For the sake of completeness, let us revisit some definitions used in Refs. [4,6].

Definition 1 (Reachability set). Consider the system given by Eq. 1. The set $\mathcal{R}\left(\xi^{*}, t\right) \subset \mathbb{R}^{n}$ is referred to as the reachability set from the point $\xi^{*}$ at time $t$ and it is the union of values at $t$ of the solutions to Eq. 1 for some admissible input function $u$ with the initial condition $\xi(0)=\xi^{*}$.

Definition 2 (Strong reachability). The system Eq. 1 is referred to as strongly reachable from the point $\xi^{*}$, if the set $\mathcal{R}\left(\xi^{*}, t\right)$ has an interior point for all $t>0$.

Definition 3 (Lie bracket). Suppose that $f \in$ $\mathbb{C}^{\infty}\left(\mathbb{R}^{n}, \mathbb{R}^{n}\right)$ and $g \in \mathbb{C}^{\infty}\left(\mathbb{R}^{n}, \mathbb{R}^{n}\right)$, then the Lie bracket of the vector fields $f$ and $g$ is

$$
[f, g]=D g f-D f g .
$$

The operator $\operatorname{ad}_{g}^{n} f: \mathbb{C}^{\infty}\left(\mathbb{R}^{n}, \mathbb{R}^{n}\right) \times \mathbb{C}^{\infty}\left(\mathbb{R}^{n}, \mathbb{R}^{n}\right) \rightarrow$ $\mathbb{C}^{\infty}\left(\mathbb{R}^{n}, \mathbb{R}^{n}\right)$ is defined as:

$$
\operatorname{ad}_{g}^{0} f=f, \quad \operatorname{ad}_{g}^{n} f=\left[g, \operatorname{ad}_{g}^{n-1} f\right] .
$$

Definition 4 (Lie algebra). Consider the vector fields $f, g \in \mathbb{C}^{\infty}\left(\mathbb{R}^{n}, \mathbb{R}^{n}\right)$. The Lie algebra generated by $f$ and $g$ is denoted by $\Lambda=\operatorname{Lie}(f, g)$ and is the smallest linear subspace of $\mathbb{C}^{\infty}\left(\mathbb{R}^{n}, \mathbb{R}^{n}\right)$ that satisfies the following conditions:

$$
\begin{aligned}
& \text { 1. } f, g \in \Lambda \text {, } \\
& \text { 2. for any } a, b \in \Lambda,[a, b] \in \Lambda \text {. }
\end{aligned}
$$

It should be noted that $\Lambda$ also defines a distribution.

Definition 5 (Distribution). The distribution $\Delta$ is the operator which assigns a linear subspace of $\mathbb{R}^{N}$ to $\forall x \in$ $\mathbb{R}^{N}$.

Definition 6 (Controllability distribution). The controllability distribution $\Delta_{c}$ of Eq. 1 is the smallest distribution which satisfies the following conditions:

1. $g \in \Delta_{c}$,

2. $\Delta_{c}$ is invariant to the vector field $f(\forall \eta \in$ $\left.\Delta_{c},[\eta, f] \in \Delta_{c}\right)$,

3. $\Delta_{c}$ is involutive $\left(\forall \eta_{1}, \eta_{2} \in \Delta_{c},\left[\eta_{1}, \eta_{2}\right] \in \Delta_{c}\right)$.

The controllability distribution has a subspace spanned by vector fields $g$ and $[f, g]$. The following theorem is a fundamental result used in Ref. [6].

Theorem 1 (Reachability rank condition). Consider the controllability distribution $\Delta_{c}$ of Eq. 1. The system Eq. 1 is strongly reachable at point $\xi^{*} \in \mathbb{R}^{n}$ if $\operatorname{dim}\{\Delta\}_{c}\left(\xi^{*}\right)=n$.

\section{Strong reachability of kinetic equations}

The active control of chemical processes may be necessary to maximize the amount of target products and minimize the amount of by-products. To achieve such a control objective, a suitable input variable must be selected so that the resulting dynamical system is controllable from that input. To check if this requirement is satisfied, the tools introduced in the previous section will be applied to the equations describing the reaction dynamics.

Consider a system of $R$ reaction steps and with $M$ species $(R, M>0)$. By borrowing notational conventions from chemistry, each reaction step can be generally defined by

$$
\begin{array}{r}
\sum_{m=1}^{M} \alpha(m, r) \mathrm{X}(m) \stackrel{k_{r}}{\longrightarrow} \sum_{m=1}^{M} \beta(m, r) \mathrm{X}(m), \\
r=1,2, \ldots, R,
\end{array}
$$

where $\alpha(\cdot, r)=(\alpha(1, r), \alpha(2, r), \ldots, \alpha(m, r))^{T}$ denotes the reactant complex vector, $\beta(\cdot, r)=$ $(\beta(1, r), \beta(2, r), \ldots, \beta(m, r))^{T}$ represents the product complex vector, $\mathrm{X}(m)$ is the $m$ th species and $k_{r}$ is the reaction rate coefficient of the $r$ th reaction step. The species on the left-hand side of Eq. 4 are referred to as reactant species and reactant complexes refer to their formal linear combinations. Similarly, one may find the products and their linear combinations (product complexes) on the right-hand side of the reaction described in Eq. 4.

Let us also define the stoichiometric matrix, denoted by $\gamma$. The matrix $\gamma$ consists of $R$ columns and $M$ rows, such that each column is obtained by

$$
\gamma(\cdot, r)=\beta(\cdot, r)-\alpha(\cdot, r)
$$

Eq. 4 defines the reaction but it does not specify its mass action kinetics. However, in order to study the controllability, the differential equations of the reaction dynamics need to be obtained in the form of differential equation Eq. 1. These equations are obtained from the heat balance [7-9] of reaction Eq. 4 as

$$
\begin{aligned}
\dot{x}_{m} & =\sum_{r=1}^{R} \gamma(m, r) k_{r} x^{\alpha(\cdot, r)} \quad m=1,2, \ldots, M, \\
\dot{T} & =\sum_{r=1}^{R} \frac{1}{\beta_{r, 0}} k_{r} x^{\alpha(\cdot, r)}+u,
\end{aligned}
$$

where $x_{m}$ denotes the concentration of species $m, T$ is the temperature, and $x^{\alpha(\cdot, r)}=\prod_{p=1}^{M} x_{p}^{\alpha(p, r)}$. Recall that the single input $u$ appears in the expression of $\dot{T}$. The state vector $\xi$ for the dynamics Eqs. 6-7 reads $\xi=\left(x_{1}, x_{2}, \ldots, x_{m}, T\right)^{T}$.

The reaction rate coefficient $k_{r}$ can be given as

$$
k_{r}=k_{r, 0} e^{-\frac{E_{r}}{R_{0} T}}
$$


where $k_{r, 0}, E_{r}, R_{0} \in \mathbb{R}^{+}$.

To study reachability, one has to determine the number of linearly independent Lie brackets spanning the Lie algebra generated by the vector fields $g$ and $\operatorname{ad}_{g} f$. Thanks to the special structure of the reaction dynamics, the linear independence can be examined by factorizing the matrix of Lie brackets and checking the rank of the factors. First, let us define the reaction dynamics matrix:

Definition 7 (Reaction dynamics matrix $D_{R}$ ). Introduce the notation

$$
k_{j}^{(i)}:=\frac{\partial^{i}}{\partial T^{i}} k_{j} \quad i, j \in\{1,2, \ldots, R\}
$$

The matrix of size $R \times R$ of the derivatives of the reaction rate coefficient defined as

$$
D_{R}=\left(\begin{array}{cccc}
k_{1}^{(1)} & k_{1}^{(2)} & \ldots & k_{1}^{(R)} \\
k_{2}^{(1)} & k_{2}^{(2)} & \ldots & k_{2}^{(R)} \\
\vdots & \vdots & \ddots & \vdots \\
k_{R}^{(1)} & k_{R}^{(2)} & \ldots & k_{R}^{(R)}
\end{array}\right)
$$

is referred to as the reaction dynamics matrix.

The following Lemma and Theorem have been shown in Ref. [4]. They are also provided here for completeness.

Lemma 1. Consider a reaction with $R$ steps. Suppose that the activation energies $E_{1}, E_{2}, \ldots, E_{R}$ of the reaction steps are all different and strictly positive, then the reaction dynamics matrix $D_{R}$ is of full rank for every $T>0$.

Theorem 1 cannot be applied directly to the reaction dynamics Eq. 6-7, because the right-hand sides (RHS) of some equations in Eq. 6 may be linearly dependent. Let $\delta$ denote the number of linearly dependent RHSs in Eq. 6. The system of chemical reactions is considered to be strongly reachable from a point $\xi^{*} \in \mathbb{R}^{M+1}$ if and only if the dimension of the controllability distribution is $M-\delta+1$. The additional dimension is due to Eq. 7 with the temperature $T$ as an additional variable.

Using Theorem 1, the controllability subspace for particular reactions can be deduced, so the conditions for strong reachability of the reactions can be determined.

Theorem 2. Consider a reaction with $M$ species and $R$ reaction steps. Suppose that the activation energies $E_{1}, E_{2}, \ldots, E_{R}$ of the reaction steps are all different and strictly positive. Then the reaction dynamics with the temperature change $(\dot{T})$ as an input variable are strongly reachable if the concentrations of all reactant species are positive.

Theorem 2 provides a condition for the strong reachability of reactions in a general form. However, for some reactions where the reaction dynamics have additional properties, weaker conditions may be sufficient to ensure strong reachability. In Sec. 4, the strong reachability conditions in the case of oximation reactions were investigated.

\section{Controllability study of the oximation re- action}

The oxidation of acetone with hydroxylamine was investigated by Raman spectroscopy in Ref. [5]. The reaction is strongly exothermic and the concentration of the intermediate highly depends on the $\mathrm{pH}$ and temperature. The process can be hazardous, however, it is not dangerous to run in a laboratory with low concentrations and in a controlled manner. Strong reachability is a necessary condition to be able to control the reaction.

\subsection{Oximation reaction in acidic medium}

In oximation reactions, the sequence (number and nature) of reaction steps depends on the $\mathrm{pH}$. The equations of the reaction steps are different in acidic and weakly basic media.

In the case of acidic media the reaction takes place over two reaction steps as given by

$$
\mathrm{NH}_{2} \mathrm{OH}+\mathrm{HCl} \stackrel{k_{1}}{\longrightarrow} \stackrel{\oplus}{\mathrm{NH}} \mathrm{H}_{3} \mathrm{OH} \stackrel{\ominus}{\mathrm{C} l}
$$

$$
\stackrel{\oplus}{\mathrm{NH}_{3} \mathrm{OH}} \stackrel{\ominus}{\mathrm{Cl}}+\stackrel{\mathrm{CH}_{3}}{\mathrm{CH}_{3}}>\mathrm{C}=\mathrm{O} \stackrel{\mathrm{k}_{2}}{\longrightarrow} \underset{\mathrm{CH}_{3}}{\mathrm{CH}_{3}}>\mathrm{C}=\stackrel{\oplus}{\mathrm{N}} \mathrm{H}-\mathrm{OH} \stackrel{\ominus}{\mathrm{Cl}}+\mathrm{H}_{2} \mathrm{O}
$$

For the sake of notational simplicity, the symbols $\mathrm{A}, \mathrm{B}, \mathrm{C}, \mathrm{D}, \mathrm{E}$, and $\mathrm{F}$ will denote the species such that the two reaction steps above read:

$$
\begin{aligned}
& \mathrm{A}+\mathrm{B} \stackrel{k_{1}}{\longrightarrow} \mathrm{C} \\
& \mathrm{C}+\mathrm{D} \stackrel{k_{2}}{\longrightarrow} \mathrm{E}+\mathrm{F} .
\end{aligned}
$$

Let us denote the concentrations of the species by $a, b, c, d, e, f \geq 0$. It is assumed that the reaction rate coefficients $k_{1}, k_{2}>0$.

Theorem 2 implies that the reaction is strongly reachable, provided that the conditions are met. It follows from strong reachability that it is possible to arrive at any concentrations of $M-\delta$ species and at any temperatures by suitable manipulation of the input. Recall that there is no guarantee that such concentrations and temperatures also define a steady-state for the system.

Note that Theorem 2 only provides a sufficient condition for strong reachability. For chemical reactions, the positivity condition of activation energies is almost always satisfied. Considering the reaction steps of the oximation reaction in acidic media the two remaining conditions of strong reachability will be studied: (1) the positivity of the concentration of all reactant species; (2) the distinctness of activation energies.

Let us now suppose that the system in Eqs. 11-12 is strongly reachable. The stoichiometric matrix for the re- 
action steps reads:

$$
\gamma=\left(\begin{array}{rr}
-1 & 0 \\
-1 & 0 \\
1 & -1 \\
0 & -1 \\
0 & 1 \\
0 & 1
\end{array}\right)
$$

and it is easy to verify that $\delta=2$ in this case. The differential equation of the reaction:

$$
\begin{aligned}
\left(\begin{array}{c}
\dot{a} \\
\dot{b} \\
\dot{c} \\
\dot{d} \\
\dot{e} \\
\dot{f} \\
\dot{T}
\end{array}\right) & =\left(\begin{array}{c}
-k_{1} a b \\
-k_{1} a b \\
k_{1} a b-k_{2} c d \\
-k_{2} c d \\
k_{2} c d \\
k_{2} c d \\
\frac{k_{1}}{\beta} a b+\frac{k_{2}}{\beta} c d
\end{array}\right)+\left(\begin{array}{l}
0 \\
0 \\
0 \\
0 \\
0 \\
0 \\
1
\end{array}\right) u= \\
& =f(\xi)+g(\xi) u
\end{aligned}
$$

is in a form similar to Eq. 1, where $\xi=$ $(a, b, c, d, e, f, T)^{T}$ and the vector field $g(\xi)$ is constant.

Since the rank of the stoichiometric matrix $\gamma$ is 2 and the temperature is a scalar quantity, Theorem 1 implies that the system is strongly reachable if $\operatorname{dim}\{\Delta\}_{c}=$ $2+1=3$. Hence, to study strong reachability, the number of linearly independent vector fields spanning the Lie algebra generated by the vector fields $\operatorname{ad}_{g} f$ and $g$ must be determined. The Lie brackets $\operatorname{ad}_{g} f$ and $\operatorname{ad}_{g}^{2} f$ read:

$$
\operatorname{ad}_{g}^{(i)} f=\left(\begin{array}{c}
-k_{1}^{(i)} a b \\
-k_{1}^{(i)} a b \\
k_{1}^{(i)} a b-k_{2}^{(i)} c d \\
-k_{2}^{(i)} c d \\
k_{2}^{(i)} c d \\
k_{2}^{(i)} c d \\
\frac{k_{1}^{(i)}}{\beta} a b+\frac{k_{2}^{(i)}}{\beta} c d
\end{array}\right)
$$

where $i \in\{1,2\}$.

To study the dimension of the controllability distribution $\Delta_{c}$ one has to determine the rank of the matrix whose columns are $g, \operatorname{ad}_{g} f$ and $\operatorname{ad}_{g}^{2} f$ which reads:

$$
\begin{aligned}
& \left(\begin{array}{ccc}
\operatorname{ad}_{g} f \quad \operatorname{ad}_{g}^{2} f \quad g
\end{array}\right)= \\
& =\left(\begin{array}{ccc}
-k_{1}^{(1)} a b & -k_{1}^{(2)} a b & 0 \\
-k_{1}^{(1)} a b & -k_{1}^{(2)} a b & 0 \\
k_{1}^{(1)} a b-k_{2}^{(1)} c d & k_{1}^{(2)} a b-k_{2}^{(2)} c d & 0 \\
-k_{2}^{(1)} c d & -k_{2}^{(2)} c d & 0 \\
k_{2}^{(1)} c d & k_{2}^{(2)} c d & 0 \\
k_{2}^{(1)} c d & k_{2}^{(2)} c d & 0 \\
\frac{k_{1}^{(1)}}{\beta} a b+\frac{k_{2}^{(1)}}{\beta} c d & \frac{k_{1}^{(2)}}{\beta} a b+\frac{k_{2}^{(2)}}{\beta} c d & 1
\end{array}\right) .
\end{aligned}
$$

The last row is linearly independent of all other rows in the matrix of Eq. 16, hence, by deleting the last row and column from the matix, the rank will be decreased by 1 . The remaining matrix is denoted by $\Theta$ and defined as

$$
\Theta=\left(\begin{array}{cc}
-k_{1}^{(1)} a b & -k_{1}^{(2)} a b \\
-k_{1}^{(1)} a b & -k_{1}^{(2)} a b \\
k_{1}^{(1)} a b-k_{2}^{(1)} c d & k_{1}^{(2)} a b-k_{2}^{(2)} c d \\
-k_{2}^{(1)} c d & -k_{2}^{(2)} c d \\
k_{2}^{(1)} c d & k_{2}^{(2)} c d \\
k_{2}^{(1)} c d & k_{2}^{(2)} c d
\end{array}\right) .
$$

It is clear that the condition $\operatorname{dim}\left\{\Delta_{c}\right\}=3$ holds true if and only if $\operatorname{rank}(\Theta)=2$. It is easy to see that the matrix $\Theta$ can be factorized as

$$
\Theta=\left(\begin{array}{cc}
-a b & 0 \\
-a b & 0 \\
a b & -c d \\
0 & -c d \\
0 & c d \\
0 & c d
\end{array}\right)\left(\begin{array}{ll}
k_{1}^{(1)} & k_{1}^{(2)} \\
k_{2}^{(1)} & k_{2}^{(2)}
\end{array}\right)=A \cdot D_{2} .
$$

The condition of $\operatorname{rank}(\Theta)=2$ can hold true if and only if the matrices $A$ and $D_{2}$ are of full rank according to the multiplication theorem of determinants. Matrix $A$ is of full rank $(\operatorname{rank}(A)=2)$ if and only if $a \neq 0, b \neq 0$, $c \neq 0$ and $d \neq 0$. The reaction dynamics matrix $D_{2}$ is of full rank if and only if there is no constant $c \in \mathbb{R} \backslash\{0\}$ such that $k_{1}^{(2)}=c k_{1}^{(1)}$ and $k_{2}^{(2)}=c k_{2}^{(1)}$, hence

$$
\frac{k_{1}^{(2)}}{k_{1}^{(1)}}=\frac{k_{2}^{(2)}}{k_{2}^{(1)}}(=c)
$$

cannot be true. Recalling that the reaction rate coefficients are $k_{r}=k_{r, 0} e^{-\frac{E_{r}}{R_{0} T}}\left(k_{r, 0}, E_{r}\right.$, and $R_{0}$ are positive constants), it is easy to determine the time derivatives:

$$
\begin{aligned}
& k_{r}^{(1)}=\left(\frac{E_{r}}{R_{0} T^{2}}\right) k_{r, 0} e^{\frac{-E_{r}}{R_{0} T}}, \\
& k_{r}^{(2)}=k_{r, 0}\left(\frac{E_{r}^{2}}{R_{0}^{2} T^{4}}-\frac{2 E_{r}}{R_{0} T^{3}}\right) e^{\frac{-E_{r}}{R_{0} T}} .
\end{aligned}
$$

The ratios of the first- and second-order time derivatives are obtained as

$$
\frac{k_{r}^{(2)}}{k_{r}^{(1)}}=\frac{k_{r, 0}\left(\frac{E_{r}^{2}}{R_{0}^{2} T^{4}}-\frac{2 E_{r}}{R_{0} T^{3}}\right) e^{\frac{-E_{r}}{R_{0} T}}}{\left(\frac{E_{r}}{R_{0} T^{2}}\right) k_{r, 0} e^{\frac{-E_{r}}{R_{0} T}}}=\frac{E_{r}-2 R_{0} T}{R_{0} T^{2}} .
$$

Based on Eq. 22, the equality in Eq. 19 holds true if and only if $E_{1}=E_{2}$.

As a result it has been proven that if the reaction dynamics are strongly reachable then the concentrations $a, b, c$ and $d$ are positive and $E_{1} \neq E_{2}$. Thus the conditions of Theorem 2 are also necessary for strong reachability in the case of oximation reactions in acidic media.

\subsection{Oximation reaction in weakly basic medium}

In the case of weakly basic media the reaction occurs according to the reaction steps given by: 


$$
\begin{aligned}
& \mathrm{NH}_{2} \mathrm{OH}+{ }_{\mathrm{CH}_{3}}^{\mathrm{CH}_{3}}>\mathrm{C}=\mathrm{O} \stackrel{\mathrm{k}_{1}}{\longrightarrow}{ }_{\mathrm{CH}_{3}}^{\mathrm{CH}_{3}}>\mathrm{C} \succ_{\mathrm{NH}-\mathrm{OH}}
\end{aligned}
$$

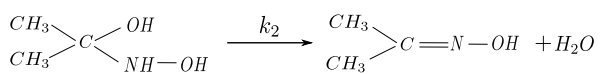

$$
\begin{aligned}
& { }_{\mathrm{CH}_{3}}^{\mathrm{CH}_{3}}>\mathrm{C}=\mathrm{N}-\mathrm{OH}+\mathrm{NaOH} \stackrel{k_{3}}{\underset{k_{-3}}{\rightleftharpoons}} \underset{\mathrm{CH}_{3}}{\mathrm{CH}_{3}}>\mathrm{C}=\mathrm{N}-\stackrel{\ominus}{\mathrm{O}} \underset{\mathrm{Na}}{\stackrel{\oplus}{\mathrm{N}}}+\mathrm{H}_{2} \mathrm{O}
\end{aligned}
$$

Since the specific chemical compositions of the species are irrelevant to the controllability analysis, the symbols A, B, C, D, E, F, and G will denote the species such that the above reaction steps read:

$$
\begin{array}{r}
\mathrm{A}+\mathrm{B} \stackrel{k_{1}}{\longrightarrow} \mathrm{C}, \\
\mathrm{C} \stackrel{k_{2}}{\longrightarrow} \mathrm{D}+\mathrm{E}, \\
\mathrm{D}+\mathrm{F} \underset{k_{-3}}{\stackrel{k_{3}}{\rightleftharpoons}} \mathrm{G}+\mathrm{E} .
\end{array}
$$

Let us denote the concentration of the species by $a, b, c$, $d, e, f, g \geq 0$. It is assumed that the reaction rate coefficients are strictly positive: $k_{1}, k_{2}, k_{3}, k_{-3}>0$.

Recall that Theorem 2 only provides a sufficient condition for strong reachability. By considering oximation reactions in weakly basic media the remaining conditions of strong reachability will be studied.

Let us suppose now that the system of Eqs. 23-25 is strongly reachable. The stoichiometric matrix for the reaction steps reads:

$$
\gamma=\left(\begin{array}{rrrr}
-1 & 0 & 0 & 0 \\
-1 & 0 & 0 & 0 \\
1 & -1 & 0 & 0 \\
0 & 1 & -1 & 1 \\
0 & 1 & 1 & -1 \\
0 & 0 & -1 & 1 \\
0 & 0 & 1 & -1
\end{array}\right)
$$

The differential equation of the reaction

$$
\begin{aligned}
\left(\begin{array}{c}
\dot{a} \\
\dot{b} \\
\dot{c} \\
\dot{d} \\
\dot{e} \\
\dot{f} \\
\dot{g} \\
\dot{T}
\end{array}\right)=\left(\begin{array}{c}
-k_{1} a b \\
-k_{1} a b \\
k_{1} a b-k_{2} c \\
k_{2} c-k_{3} d f+k_{-3} g e \\
k_{2} c+k_{3} d f-k_{-3} g e \\
-k_{3} d f+k_{-3} g e \\
k_{3} d f-k_{-3} g e \\
\frac{k_{1}}{\beta} a b+\frac{k_{2}}{\beta} c+\frac{k_{3}}{\beta} d f+\frac{k_{-3}}{\beta} g e
\end{array}\right)+ \\
+\left(\begin{array}{c}
0 \\
0 \\
0 \\
0 \\
0 \\
0 \\
0 \\
1
\end{array}\right) u=f(\xi)+g(\xi) u
\end{aligned}
$$

is in a form similar to Eq. 1, where the vector field $g(\xi)$ is constant and $\xi=(a, b, c, d, e, f, g, T)^{T}$.
Since the rank of the stoichiometric matrix $\gamma$ is 3 and the temperature is a scalar quantity, Theorem 1 implies that the system is strongly reachable if $\operatorname{dim}\{\Delta\}_{c}=3+$ $1=4$. Hence, the number of linearly independent vector fields spanning the Lie algebra generated by the vector fields $\operatorname{ad}_{g} f$ and $g$ must be determined.

The Lie-brackets $\operatorname{ad}_{g} f, \operatorname{ad}_{g}^{2} f$ and $\operatorname{ad}_{g}^{3} f$ read:

$$
\operatorname{ad}_{g}^{i} f=\left(\begin{array}{c}
-k_{1}^{(i)} a b \\
-k_{1}^{(i)} a b \\
k_{1}^{(i)} a b-k_{2}^{(i)} c \\
k_{2}^{(i)} c-k_{3}^{(i)} d f+k_{-3}^{(i)} g e \\
k_{2}^{(i)} c+k_{3}^{(i)} d f-k_{-3}^{(i)} g e \\
-k_{3}^{(i)} d f+k_{-3}^{(i)} g e \\
k_{3}^{(i)} d f-k_{-3}^{(i)} g e \\
\frac{k_{1}^{(i)}}{\beta} a b+\frac{k_{2}^{(i)}}{\beta} c+\frac{k_{3}^{(i)}}{\beta} d f+\frac{k_{-3}^{(i)}}{\beta} g e
\end{array}\right),
$$

where $i \in\{1,2,3\}$. To study the dimensions of the controllability distribution $\Delta_{c}$ one has to give the rank of the matrix whose columns are $\operatorname{ad}_{g} f, \operatorname{ad}_{g}^{2} f, \operatorname{ad}_{g}^{3} f$, and $g$ :

$$
\left(\operatorname{ad}_{g} f \quad \operatorname{ad}_{g}^{2} f \quad \operatorname{ad}_{g}^{3} f \quad g\right) .
$$

The last row is linearly independent of all other rows in matrix Eq. 30, hence, by deleting the last row and column from the matrix, the rank will be decreased by 1 . The remaining matrix is denoted by $\Theta$ and defined as

$$
\Theta=\left\{\left(\begin{array}{c}
-k_{1}^{(i)} a b \\
-k_{1}^{(i)} a b \\
k_{1}^{(i)} a b-k_{2}^{(i)} c \\
k_{2}^{(i)} c-k_{3}^{(i)} d f+k_{-3}^{(i)} g e \\
k_{2}^{(i)} c+k_{3}^{(i)} d f-k_{-3}^{(i)} g e \\
-k_{3}^{(i)} d f+k_{-3}^{(i)} g e \\
k_{3}^{(i)} d f-k_{-3}^{(i)} g e
\end{array}\right)_{i=1,2,3}\right\}
$$

The condition $\operatorname{dim}\{\Delta\}_{c}=4$ holds true if and only if $\operatorname{rank}(\Theta)=3$. It is easy to see that the matrix $\Theta$ can be factorized as

$$
\begin{aligned}
& \Theta=A \cdot D= \\
& \left(\begin{array}{cccc}
-a b & 0 & 0 & 0 \\
-a b & 0 & 0 & 0 \\
a b & -c & 0 & 0 \\
0 & c & -d f & g e \\
0 & c & d f & -g e \\
0 & 0 & -d f & g e \\
0 & 0 & d f & -g e
\end{array}\right)\left(\begin{array}{ccc}
k_{1}^{(1)} & k_{1}^{(2)} & k_{1}^{(3)} \\
k_{2}^{(1)} & k_{2}^{(2)} & k_{2}^{(3)} \\
k_{3}^{(1)} & k_{3}^{(2)} & k_{3}^{(3)} \\
k_{-3}^{(1)} & k_{-3}^{(2)} & k_{-3}^{(3)}
\end{array}\right)
\end{aligned}
$$

The condition $\operatorname{rank}(\Theta)=3$ can hold true only if $\operatorname{rank}(A) \geq 3$ and $\operatorname{rank}(D) \geq 3$. $\mathrm{T}$ he $3 \mathrm{rd}$ and 4 th columns in matrix $A$ are linearly dependent, thus $\operatorname{rank}(A) \leq 3$. The condition $\operatorname{rank}(A)=3$ can hold true only if the concentrations $a, b$, and $c$ as well as the 
concentrations $d$ and $f$, or the concentrations $g$ and $e$ are strictly positive. Matrix $D$ is of full rank only if $D$ consists of a $3 \times 3$ times full-rank matrix. Lemma 1 implies that the reaction dynamics matrix $D_{3}$ is of full rank if the activation energies are different. Hence, matrix $D$ is of full rank, if 3 different activation energies exist.

The system was proven to be strongly reachable if 3 of the activation energies are all different and concentrations $a, b, c$ and $d, f$, or $g, e$ are positive. Thus a condition for strong reachability coule be given more precisely in the case of oximation reaction in weakly basic media.

\section{Reversible reaction step}

For reactions of general types, Theorem 2 provides a condition for strong reachability. However, it will be shown that if the reaction contains one or more reversible steps, weaker conditions are sufficient for strong reachability.

The concentrations are defined by $x_{1}, x_{2}, \ldots, x_{M}$, as in the previous sections. The notation $\mathcal{A}$ is introduced for the matrix describing the effect of concentrations:

$$
\mathcal{A}:=\gamma \operatorname{diag}\left(x^{\alpha(\cdot, 1)}, x^{\alpha(\cdot, 2)}, \ldots, x^{\alpha(\cdot, R)}\right),
$$

where $x^{\alpha(\cdot, r)}=\prod_{m=1}^{M} x_{m}^{\alpha(m, r)}$ and $\gamma$ is the stoichiometric matrix as introduced by Eq. 5. The vector $k=$ $\left(k_{1}, k_{2}, \ldots, k_{R}\right)^{T}$ is composed of the reaction rate coefficients. The notation $\mathcal{D}$ is introduced for the following matrix composed of the derivatives of reaction rate coefficients:

$$
\mathcal{D}:=\left(k^{(1)} k^{(2)} \ldots k^{(\operatorname{rank}(\gamma))}\right) .
$$

Lemma 2. The reaction dynamics in Eqs. 6-7 with the input variable $\dot{T}$ are strongly reachable, if $\operatorname{rank} \gamma=$ $\operatorname{rank}(\mathcal{A D})$, where $\gamma$ is the stoichiometric matrix and $\mathcal{A}$ and $\mathcal{D}$ are defined as above.

Proof The differential equation of the reaction reads:

$$
\left(\begin{array}{c}
\dot{x} \\
\dot{T}
\end{array}\right)=\left(\sum_{r=1}^{R} \frac{k_{r}}{\beta_{r, 0}} x^{\alpha(\cdot, r)}\right)+\left(\begin{array}{l}
0 \\
1
\end{array}\right) u,
$$

where $\dot{x}=\left(\dot{x}_{1}, \dot{x}_{2}, \ldots, \dot{x}_{M}\right)^{T}, u$ is the control input and the vector field $v$ stands for the vector composed of the right-hand sides of Eq. 6 .

As in the previous sections, the study of strong reachability means verification of the dimension of the controllability distribution $\Delta_{c}$. The dimension of the controlled input (the dimension of the change in temperature) is 1 , thus, Theorem 1 implies that the system is strongly reachable if and only if $\operatorname{dim}\{\Delta\}_{c}=\operatorname{rank}(\gamma)+1$.

The vector fields spanning the controllability distribution are $g$ and $\operatorname{ad}_{g}^{i} f$ for $i>0$. The Lie bracket $\operatorname{ad}_{g}^{i} f$ reads:

$$
\operatorname{ad}_{g}^{i} f=\left(\begin{array}{c}
v^{(i)} \\
\sum_{r=1}^{R} \frac{k_{r}^{(i)}}{\beta_{r, 0}} x^{\alpha(\cdot, r)}
\end{array}\right)=\left(\begin{array}{c}
\mathcal{A} \cdot k^{(i)} \\
\sum_{r=1}^{R} \frac{k_{r}^{(i)}}{\beta_{r, 0}} x^{\alpha(\cdot, r)}
\end{array}\right)
$$

for $i \in\{1,2, \ldots, \operatorname{rank} \gamma\}$. The rank of the controllability distribution is hence the rank of the matrix

$$
\begin{aligned}
& \left(\begin{array}{lllll}
\operatorname{ad}_{g} f & \operatorname{ad}_{g}^{2} f & \ldots & \operatorname{ad}_{g}^{\operatorname{rank} \gamma} f & g
\end{array}\right)= \\
& \left(\begin{array}{cccc}
\mathcal{A} \cdot k^{(1)} & \ldots & \mathcal{A} \cdot k^{(\operatorname{rank} \gamma)} & 0 \\
\sum_{r=1}^{R} \frac{k_{r}^{(1)}}{\beta_{r, 0}} x^{\alpha(\cdot, r)} & \ldots & \sum_{r=1}^{R} \frac{k^{(\operatorname{rank} \gamma)}}{\beta_{r, 0}} x^{\alpha(\cdot, r)} & 1
\end{array}\right)
\end{aligned}
$$

The last row in Eq. 36 is linearly independent of the others, hence, the same reasoning as earlier is followed and the last row and columns are eliminated, thus, decreasing the rank by one. The resulting matrix is denoted by $\Theta$ and reads:

$$
\begin{array}{r}
\Theta=\left(\begin{array}{rr}
\mathcal{A} \cdot k^{(1)} & \mathcal{A} \cdot k^{(2)} \ldots \mathcal{A} \cdot k^{(\mathrm{rank} \gamma)}
\end{array}\right)= \\
=\mathcal{A D} .
\end{array}
$$

Since the dimension of the controllability distribution is $\operatorname{rank}(\Theta)+1$, the reaction dynamics are strongly reachable if $\operatorname{rank} \gamma=\operatorname{rank} \Theta$ or if $\operatorname{rank} \gamma=\operatorname{rank}(\mathcal{A D})$.

Theorem 3. Consider the reaction dynamics Eqs. 6-7 such that the activation energies $E_{1}, E_{2}, \ldots, E_{R}$ are positive and different in pairs. Suppose that the concentrations of reactant species are positive in the case of oneway reaction steps and at least one of the ways is positive in the case of reversible reaction steps. Then, the reaction dynamics controlled by $\dot{T}$ are strongly reachable.

Proof If the system does not contain reversible reaction steps, Theorem 2 is obtained.

Without loss of generality, it can be supposed that the system contains one reversible reaction step. This step can be replaced by pairs of irreversible reaction steps, with reaction rate coefficients denoted by $k_{e}$ and $k_{-e}$. The changes in the concentrations are equal in the reaction step with rate coefficient $k_{e}$ and in the reaction step with rate coefficient $k_{-e}$, only the direction is different. Thus, the two columns in matrix $\gamma$ for the reversible reaction steps are always linearly dependent. Lemma 2 implies that the system is strongly reachable if and only if $\operatorname{rank} \gamma=\operatorname{rank}(\mathcal{A D})$. If the activation energies are positive and all different, Lemma 1 implies that matrix $\mathcal{D}$ is of full rank. The matrix $\mathcal{A}$ is defined by Eq. 32, thus, the columns for $k_{e}$ and $k_{-e}$ are linearly dependent. The column for $k_{e}$ contains the factor of the concentrations of the reactant species in the transformation step and $k_{-e}$ contains the factor of the concentrations of the reactant species in the transformation step in the opposite direction with the arbitrary sign in the place of non-zero elements. By substituting one of the two vector fields with a zero vector field, the rank of matrix $\mathcal{A}$ remains unchanged. Thus, in the case of reversible reactions for strong reachability, it is sufficient if the reactant species have positive concentrations only in one of the directions, and the activation energies are positive and all different. 


\section{Conclusion}

The reaction dynamics of strong reachability where the control variable is selected as the rate of change in the ambient temperature $(\dot{T})$ have been studied. First, the strong reachability was analyzed in the case of the oximation reaction. Since the processes depend on the $\mathrm{pH}$, conditions that facilitate strong reachability in acidic as well as weakly basic media were studied. For our analysis, Theorem 2 was used. It provides sufficient conditions to facilitate strong reachability, however, these conditions are not always necessary. It was proven that the conditions in Theorem 2 are necessary to facilitate strong reachability of the oximation reaction in the case of acidic media. In weakly basic media, the system contained a reversible reaction step, thus, the conditions of Theorem 2 could be determined.

Strong reachability has also been studied for reaction dynamics of a general type that contain at least one reversible reaction step where the conditions of Theorem 2 could also be further refined. For reversible reaction steps it has been shown that positive reactant concentrations are unnecessary to facilitate strong reachability in both directions of the reversible steps, in one direction is sufficient.

\section{Acknowledgement}

The chemistry-related comments from Zsombor Kristóf Nagy and György Marosi at the BME Department of Organic Chemistry and Technology are gratefully acknowledged.

\section{REFERENCES}

[1] Farkas, G.: Local controllability of reactions, J. Math. Chem., 1998 24, 1-14 DOI: 10.1023/A:1019150014783

[2] Drexler, D.A., Tóth, J.: Global controllability of chemical reactions, J. Math. Chem., 2016 54, 13271350 DOI: 10.1007/s10910-016-0626-7

[3] Dochain, D., Chen, L.: Local observability and controllability of stirred tank reactors, J. Math. Chem., 1992 2, 139-144 DOI: 10.1016/0959-1524(92)85003-F

[4] Drexler, D.A., Virágh, E., Tóth, J.: Controllability and reachability of reactions with temperature and inflow control, Fuel, 2017 211, 906-911 DOI: 10.1016/j.fuel.2017.09.095

[5] Csontos, I., Pataki, H., Farkas, A., Bata, H., Vajna, B., Nagy, Z.K., Keglevich, G., Marosi, G.J.: Feedback Control of Oximation Reaction by Inline Raman Spectroscopy, Organic Process Research \& Development, 2014 19, 189-195 DOI: 10.1021/op500015d

[6] Isidori, A.: Nonlinear Control System (Springer Verlag, London), 1995 DOI: 10.1007/978-1-84628-615-5

[7] Érdi, P., Tóth, J.: Mathematical models of chemical reactions. Theory and applications of deterministic and stochastic models (Princeton University Press, Princeton, New Jersey), 1989 ISBN: 9780719022081

[8] Turányi, T., Tomlin, A.S.: Analysis of Kinetic Reaction Mechanisms (Springer Berlin Heidelberg), 2014 ISBN: 9783662445624

[9] Atkins, P.W.: Physical Chemistry (Oxford University Press), 2010 ISBN: 9780199543373 